\title{
Seeing in the Dark
}

Improving Understanding of Driver Visibility Requirements at Night

Exploratory Advanced Research ... Next Generation Transportation Solutions

$\mathbf{W}$ hat visual cues aid drivers the most as they drive at night? Advancing knowledge and understanding of how drivers acquire and act on visual information while driving at night is the goal of "Increased Understanding of Driver Visibility Requirements," an Exploratory Advanced Research (EAR) Program project launched by the Federal Highway Administration (FHWA) in 2008.

\section{Determining the Quantity and} Quality of Visual Information Needed

The project will develop a theoretical framework for determining the quantity and quality of visual information needed by drivers to safely navigate the roadway, focusing on two-lane rural roads.

One-third of all roadway fatalities involve vehicles that run off the road, with this statistic increasing to two-thirds of fatalities in rural settings. While daytime driving has been analyzed in numerous human studies and in computer model development, much less is known about how drivers function at night.

The project will investigate how both human drivers and autonomous robotic vehicles negotiate curves at night, with the aim of developing a software program that can predict how both human drivers and autonomous vehicle systems will respond to different combinations of visual guidance to navigate curves in the roadway. This visual guidance can range from centerlines and edge lines to delineators and raised pavement markers. The software program will allow researchers to better assess the usefulness of the visual guidance provided to drivers. As a starting point, the project will use the Four Dimensional Real-Time Control System (4D/RCS) methodology developed by the National Institute of Standards and Technology (NIST) for the control of autonomous on-road vehicles.

\section{Developing a Revolutionary Design Tool}

"Rather than measuring how well drivers perform, we want to evaluate how drivers acquire visual information and how they then use that information," says Carl Andersen at FHWA's Turner-Fairbank Highway Research Center.

The resulting software program could be used to evaluate roadways, identify possible problem areas, and improve roadway safety. This revolutionary design tool would significantly advance the understanding of how drivers acquire and act on visual information while driving at night and could assist FHWA and other transportation agencies with determining how to best design roadways to meet driver needs. In the long term, the research could also advance the vision of a future highway system that incorporates autonomous vehicles traveling under supervisory human control.

\section{Progress}

The project is being conducted by Science Applications International Corporation (SAIC), the Texas Transportation Institute (TTI), and NIST. Driver performance under varying levels of pavement marking luminance was measured at TTI in summer 2008. Data from this initial study is being used to refine the cognitive task analysis for the autonomous vehicle. It is anticipated that the autonomous vehicle will drive the test course at TTI in April 2009 under the same conditions as those experienced by the human participants, with a final report on the project due in late 2009. 


\section{Seeing in the Dark}

Improving Understanding of Driver Visibility Requirements at Night

\section{Future Efforts}

The results of the autonomous vehicle driving tests are key to determining the feasibility of developing the desired model of human perception. Developing a robotic system based on the NIST 4D/RCS methodology that performs similarly to people when carrying out various driving tasks will provide insights into the processes by which people acquire the visual information needed to drive. This will, in turn, allow researchers to develop models to assess the adequacy of the visual information available to drivers.

\section{Learn More}

For more information on the project, contact Carl Andersen at FHWA, 202-493-3366 (email: carl.andersen@fhwa.dot.gov).

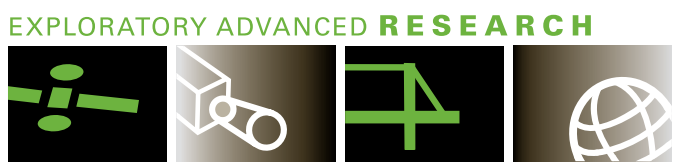

\section{What Is the Exploratory Advanced Research Program?}

FHWA's Exploratory Advanced Research (EAR) Program focuses on long-term, high-risk research with a high payoff potential. The program addresses underlying gaps faced by applied highway research programs, anticipates emerging issues with national implications, and reflects broad transportation industry goals and objectives.

To learn more about the EAR Program, visit the Exploratory Advanced Research Web site at www.fhwa.dot.gov/ advancedresearch. The site features information on research solicitations, updates on ongoing research, links to published materials, summaries of past EAR Program events, and details on upcoming events. For additional information, contact David Kuehn at FHWA, 202-493-3414 (email: david.kuehn@fhwa.dot.gov), or Terry Halkyard at FHWA, 202-493-3467 (email: terry.halkyard@fhwa.dot.gov). 